\title{
Rapid Identification of Antifungal Compounds against Exserohilum rostratum Using High Throughput Drug Repurposing Screens
}

\author{
Wei Sun ${ }^{19}$, Yoon-Dong Park ${ }^{29}$, Janyce A. Sugui ${ }^{2}$, Annette Fothergill ${ }^{3}$, Noel Southall ${ }^{1}$, Paul Shinn ${ }^{1}$, \\ John C. McKew ${ }^{1}$, Kyung J. Kwon-Chung ${ }^{2}$, Wei Zheng ${ }^{1 *}$, Peter R. Williamson ${ }^{2,4 *}$
}

1 National Center for Advancing Translational Sciences, National Institutes of Health, Bethesda, Maryland, United States of America, 2 Laboratory of Clinical Infectious Diseases, National Institute of Allergy and Infectious Diseases, National Institutes of Health, Bethesda, Maryland, United States of America, $\mathbf{3}$ University of Texas Health Science Center, San Antonio, Texas, United States of America, $\mathbf{4}$ Section of Infectious Diseases, Immunology and International Medicine, University of Illinois College of Medicine, Chicago, Illinois, United States of America

\begin{abstract}
A recent large outbreak of fungal infections by Exserohilum rostratum from contaminated compounding solutions has highlighted the need to rapidly screen available pharmaceuticals that could be useful in therapy. The present study utilized two newly-developed high throughput assays to screen approved drugs and pharmaceutically active compounds for identification of potential antifungal agents. Several known drugs were found that have potent effects against $E$. rostratum including the triazole antifungal posaconazole. Posaconazole is likely to be effective against infections involving septic joints and may provide an alternative for refractory central nervous system infections. The anti-E. rostratum activities of several other drugs including bithionol (an anti-parasitic drug), tacrolimus (an immunosuppressive agent) and floxuridine (an antimetabolite) were also identified from the drug repurposing screens. In addition, activities of other potential antifungal agents against $E$. rostratum were excluded, which may avoid unnecessary therapeutic trials and reveals the limited therapeutic alternatives for this outbreak. In summary, this study has demonstrated that drug repurposing screens can be quickly conducted within a useful time-frame. This would allow clinical implementation of identified alternative therapeutics and should be considered as part of the initial public health response to new outbreaks or rapidly-emerging microbial pathogens.
\end{abstract}

Citation: Sun W, Park Y-D, Sugui JA, Fothergill A, Southall N, et al. (2013) Rapid Identification of Antifungal Compounds against Exserohilum rostratum Using High Throughput Drug Repurposing Screens. PLoS ONE 8(8): e70506. doi:10.1371/journal.pone.0070506

Editor: Ping Wang, Research Institute for Children and the Louisiana State University Health Sciences Center, United States of America

Received April 16, 2013; Accepted June 18, 2013; Published August 21, 2013

This is an open-access article, free of all copyright, and may be freely reproduced, distributed, transmitted, modified, built upon, or otherwise used by anyone for any lawful purpose. The work is made available under the Creative Commons CCO public domain dedication.

Funding: This work was supported by the Intramural Research Programs of the National Center for Advancing Translational Sciences and National Institute of Allergy and Infectious Diseases, National Institutes of Health. The funders had no role in study design, data collection and analysis, decision to publish, or preparation of the manuscript.

Competing Interests: The authors have declared that no competing interests exist.

*E-mail: williamsonpr@mail.nih.gov (PRW); wzheng@mail.nih.gov (WZ)

9 These authors contributed equally to this work.

\section{Introduction}

Unusual or highly antibiotic resistant organisms may subject large numbers of individuals to unexpected infectious diseases due to greater globalization that brings more widespread distribution networks and potential threats such as bioterrorism. Limited therapeutic options or failures in conventional therapy during these outbreaks can be encountered because of either intolerable drug toxicities or lack of efficacious drugs. Recently, a large outbreak of fungal infections has been caused by the widespread distribution of contaminated preservative-free methylprednisolone acetate prepared by a single compounding pharmacy $[1,2,3,4]$. It has currently resulted in 741 infections with 55 deaths [5]. Exserohilum rostratum, a dermatiaceous fungus commonly found on plants, in soil and in households, has been identified as one of the predominant pathogens in the current multistate outbreak of fungal meningitis and other infections associated with contaminated steroid injections. Although it rarely causes infections in healthy people, infections of skin and corneal tissues as well as more disseminated infections in immunocompromised populations have been reported $[6,7,8]$.

E. rostratum is sensitive to amphotericin B, a commonly used antifungal agent, but the severe and potentially lethal side-effects of this drug have limited its use in certain patients. While traditional antibiotic susceptibility testing has provided initial recommendations of using amphotericin B for treatment, the advanced age (median 69) of the patient population in this outbreak has limited the therapeutic efficacy in many patients, mainly due to drug toxicity. There are few alternative drugs that are known for the treatment of infections caused by E. rostratum. The conventional process for drug discovery and development cannot accommodate such a sudden outbreak as it requires 10-12 years in average to develop a drug. Recently, a drug repurposing screen with approved drugs and pharmacologically active agents [9] has emerged as an alternative approach to rapidly identify new therapeutic indications that have been successfully applied to several diseases $[10,11,12,13,14,15]$. This unique approach may also help to quickly identify alternative therapeutics for the 
Table 1. Assay protocol for the measurements of fungicidal compounds.

\begin{tabular}{llll}
\hline & & & \\
\hline Step & Parameter & Value & Description \\
\hline 1 & PBS/Medium & $2.5 \mu \mathrm{l} /$ well & PBS or RPMI Medium \\
2 & Compound & $0.023 \mu \mathrm{l} /$ well & Compound in DMSO solution \\
3 & Hyphae/Conidia & $2.5 \mu \mathrm{l} /$ well & Hyphae in PBS or Conidia in RPMI Medium \\
4 & Incubation & $24 \mathrm{hr}$ & $37^{\circ} \mathrm{C}, 5 \% \mathrm{CO}_{2}$ \\
5 & Detection reagent & $4 \mu \mathrm{l} /$ well & ATP content assay reagent \\
6 & Incubation & 15 min & Room temperature \\
7 & Plate reading & Luminescence mode & ViewLux plate reader \\
\hline
\end{tabular}

Note: PBS was dispensed in the hyphae assay following by transferring of compound and addition of hyphae cell suspension. RPMI Medium, compound and conidia cells in RPMI Medium were sequentially added in the conidia assays.

doi:10.1371/journal.pone.0070506.t001

treatment of infections caused by outbreaks such as that by $E$. rostratum.

Here we report the development and optimization of two assays using E. rostratum hyphae and conidia in an ATP content assay format for high throughput screening. Both assays were screened in parallel against two known compound libraries including 4096 approved drugs and 1280 compounds with pharmacologically known activities. Within seven weeks, the activities of 20 known antifungals, 8 other anti-infectious agents and 10 other drugs against $E$. rostratum were identified from the screens. While some of these drugs may be considered as alternative therapeutics to treat E. rostratum infections, others could serve as tools for identification of new molecular targets for future drug development.

\section{Materials and Methods}

\section{Materials}

Amphotericin B (catalog \# A9528) was purchased from SigmaAldrich (St. Louis, MO). The ATP content kit (ATPlite, catalog No. 6016941) was purchased from PerkinElmer (Waltham, MA). PBS (Catalog No. 10010049) was purchased from Life technologies. The 1536-well white sterile tissue culture treated polystyrene plates (Catalog No. 789092-F) were purchased from Greiner BioOne (Monroe, NC).

\section{Preparation of Exserohilum rostratum conidia and hyphal fragments}

Conidia and hyphae of E. rostratum were obtained as described by Richard et al. [16], with the following modifications. Briefly, conidia were harvested from Potato Dextrose Agar (PDA) cultured media with $0.05 \%$ Tween 80 , and the conidial suspension was filtered using a Cell Strainer (100 $\mu \mathrm{m}$, BD Falcon REF 352340). After centrifugation at $700 \mathrm{~g}$ for $10 \mathrm{~min}$, the suspension was decanted and conidia were resuspended at $1 \times 10^{5}$ per $\mathrm{ml}$ in RPMI and counted in a hemocytometer. Hyphae were harvested from yeast extract peptone dextrose (YPD) culture media with $0.05 \%$ Tween 80 . Hyphal fragments were sized by vortexing $15 \mathrm{sec}$ twice with $0.4 \mathrm{~mm}$ glassbeads, and the hyphae suspension was filtered by cheese cloth twice. Microscopy was used to determine the size of hyphal fragments, which ranged between $10-50 \mu \mathrm{m}$. To normalize concentrations of hyphal fragments for batch to batch consistency, carbohydrate analysis was performed by a phenolsulfuric acid method as previously described [17]. The final stock concentration of hyphae was adjusted to $1.0\left(\mathrm{OD}_{490}\right)$ per $100 \mu \mathrm{l}$.

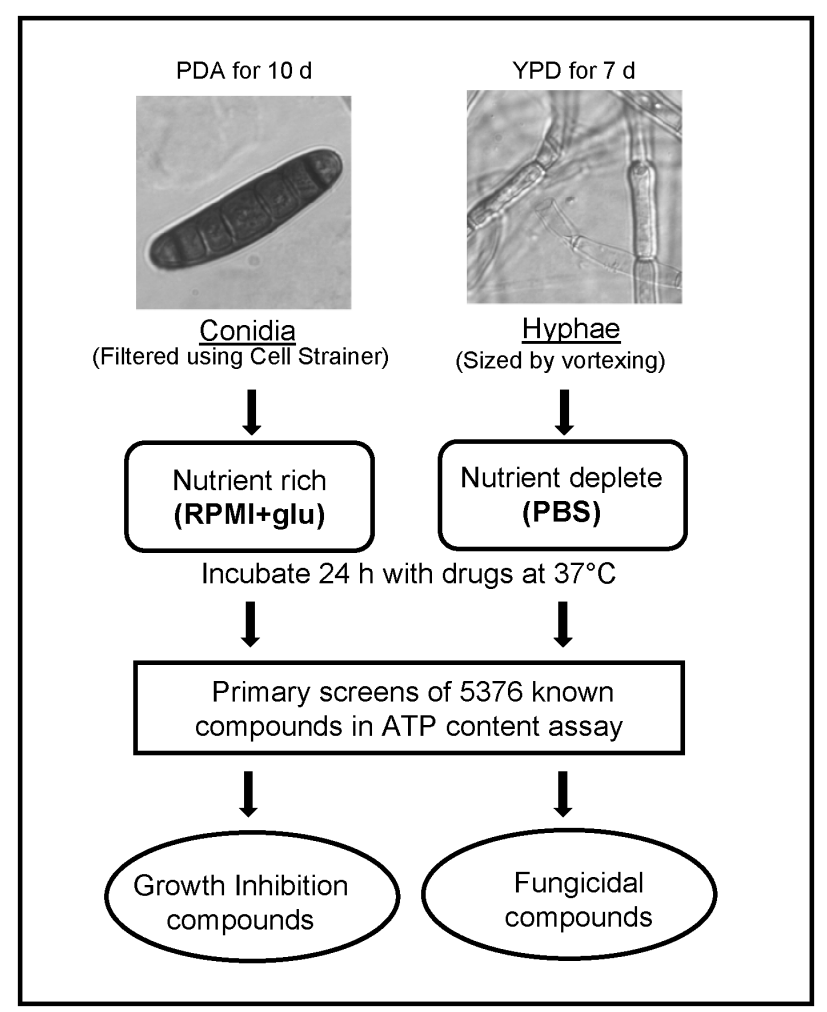

Figure 1. Scheme of hyphae and conidia assay methodology. Conidia harvested from colonies grown on potato dextrose agar (PDA) were incubated in nutrient-rich RPMI media with compounds to identify inhibitors of $E$. rostratum growth. Alternatively, hyphal fragments, obtained by growth on Yeast Extract Peptone Dextrose agar (YPD) and size-reduced by sonication, were incubated with compounds under nutrient-deprived conditions to identify fungicidal compounds. doi:10.1371/journal.pone.0070506.g001

\section{Mammalian cell culture}

Human neuroblastoma SH-SY5Y cell line (Catalog No. CRL2266) was purchased from ATCG (Manassas, VA). SH-SY5Y cell line was cultured in $175-\mathrm{cm}^{2}$ tissue culture flasks (Costar, Cambridge, MA) with $30 \mathrm{ml}$ of growth medium at $37^{\circ} \mathrm{C}$ in a $5 \% \mathrm{CO}_{2}$ humidified atmosphere. Growth medium was made with Dulbecco's Modified Eagle Medium: Nutrient Mixture F-12 with $10 \%$ fetal bovine serum (FBS). Growth medium was replaced every other day and cells were passed at $75 \%$ confluence. 
A. Cell viability

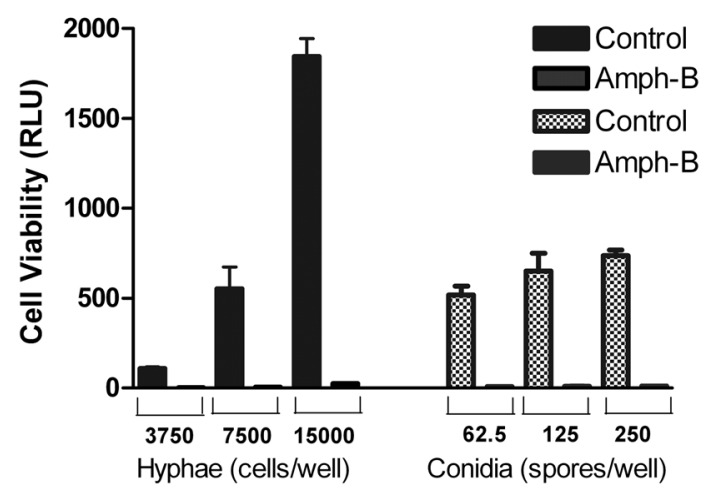

B. S/B ratio

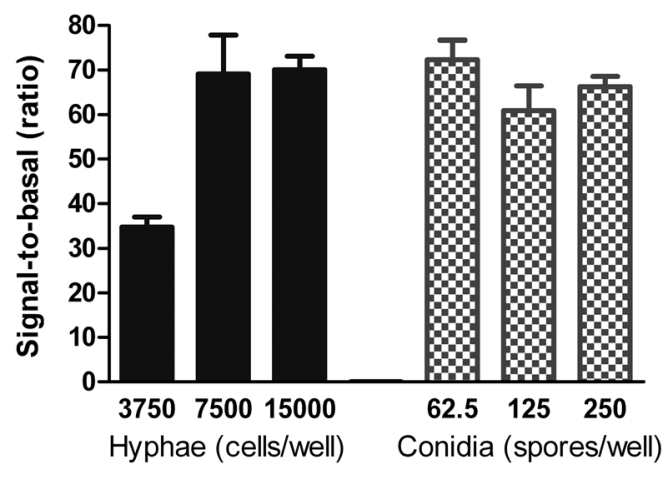

Figure 2. E. rostratum cell density optimization in a 1536-well plate assay format. (A) The viability of hyphal fragments was assayed at 3750 , 7500 and 15000 fragments/well suspended in PBS after incubation with $46 \mu \mathrm{M}$ amphotericin B (Amph-B) or DMSO (solvent control) for $24 \mathrm{~h}$ at $37^{\circ} \mathrm{C}$ using the ATP content assay. Similarly, the viability of conidia (spores) was assayed at $62.5,125$ and 250 cells/well in nutrition rich RPMI medium. (B) Signal-to-basal ratios of the hyphal fragment and conidia assays in different cell densities. The total signal and basal signal were defined by the cells treated with DMSO and $46 \mu \mathrm{M}$ Amph-B, respectively. (RLU: relative luminescence units).

doi:10.1371/journal.pone.0070506.g002

\section{ATP content assay}

The ATPLite assay kit, consisting of luciferase/luciferin and detergent, was used to quantitate cellular ATP levels as a marker for cell viability (Table 1). For assay development, hyphal fragments or conidia in PBS were plated in a seeding density of 3,750, 7,500 and 15,000 cellular particles/well with a final volume of $5 \mu \mathrm{l} /$ well using the Multidrop-Combi dispenser in white 1536well plates. Conidia or hyphae fragments were incubated for $24 \mathrm{~h}$ at $37^{\circ} \mathrm{C}$ in a $5 \% \mathrm{CO}_{2}$ humidified atmosphere. ATPLite detection reagents were added at $4 \mu \mathrm{l} /$ well. The assay plates were incubated at room temperature for 15 minutes before reading luminescence intensity on a ViewLux plate reader (Perkin Elmer, Waltham, MA). Conidia suspended in RPMI medium were inoculated at concentrations of $62.5,125$ and 250 conidia/well with a final volume of $5 \mu \mathrm{l} /$ well in white 1536 -well plates. The conidia assay was developed and optimized in the same way as the hyphal assay.

\section{Compound library and liquid handling instrument}

The library of pharmacologically active compounds (LOPAC) consists of 1280 small molecules with characterized biological activities. The LOPAC library (Sigma-Aldrich) has been extensively used for HTS assay validations [10,18]. The NIH Chemical
Genomics Center Pharmaceutical (NPC) collection was constructed in-house through a combined effort of compound purchasing and custom synthesis [9] and recently expanded to 4096 compounds. In this drug libarary, $52 \%$ of which are drugs approved for human or animal use by the United States Food and Drug Administration (FDA), 22\% are drugs approved in Europe, Canada, or Japan, and the remaining $25 \%$ are compounds that have entered clinical trials or are research compounds commonly used in biomedical research. Compounds from both libraries were obtained as powder samples and dissolved in DMSO as $10 \mathrm{mM}$ stock solutions, except several hundred from the NPG library that were prepared as $4.47 \mathrm{mM}$ stock solutions due to solubility limitations. 2.5 $\mu \mathrm{l}$ /well PBS was dispensed into 1536-well plates using a Multidrop-Combi dispenser. Compound in DMSO solution was transferred in a volume of $23 \mathrm{nl} /$ well using a NXTR pintool station (WAKO Scientific Solutions, San Diego, CA). This additional PBS step for compound addition was designed to prevent the potential fungal contamination of compound libraries. The hyphal fragments in PBS were then added at $2.5 \mu \mathrm{l} /$ well for a final seeding density of 15,000 hyphal fragments/well using the Multidrop-Combi dispenser. The final compound concentration was $46 \mu \mathrm{M}$ in the primary screen. The assay plates were incubated

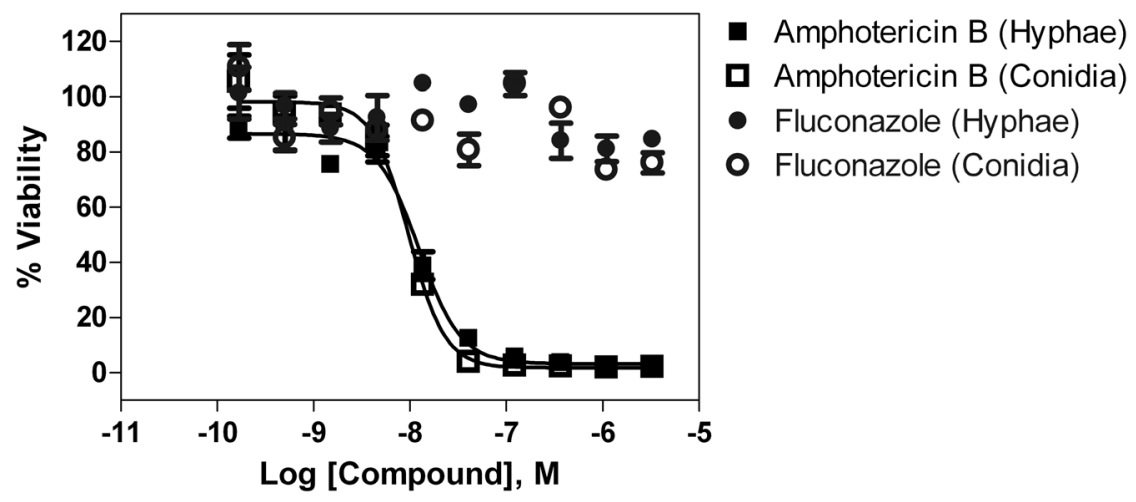

Figure 3. Concentration-response curves of Amphotericin B and fluconazole against $E$. rostratum in hyphal fragment and conidia assays. The $\mathrm{IC}_{50}$ values of amphotericin B in the hyphae assay with 15000 fragments/well and conidia assay with 250 cells/well were $12.4 \mathrm{nM}$ (9.93 to $15.6 \mathrm{nM}, 95 \%$ confidence intervals) and $9.71 \mathrm{nM}$ (8.24 to $11.4 \mathrm{nM}$ ), respectively. Fluconazole showed no activity in both assays. doi:10.1371/journal.pone.0070506.g003 


\section{Primary Screens in hyphae and conidia assays}

(1280 compounds with known activities +4096 compounds in an approved drug library)

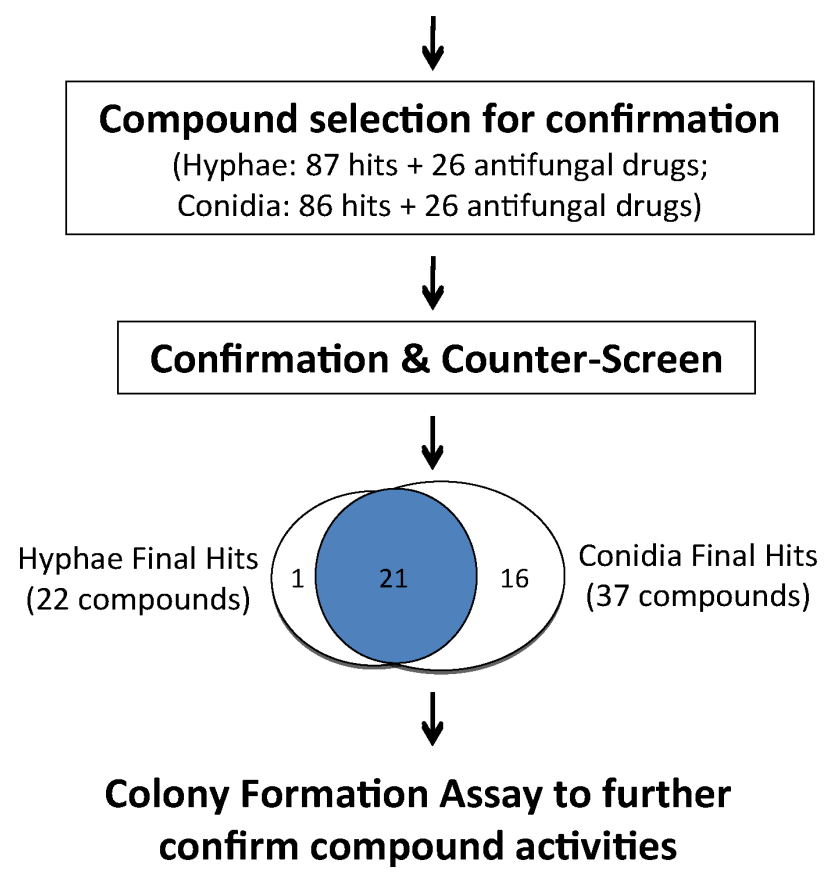

Figure 4. Flowchart of $E$. rostratum viability screens and compound confirmation in hyphal fragment (left) and conidia (right) assays. The primary screens of the LOPAC and approved drug libraries were carried out in the hyphal fragment and conidia assays. A group of 87 hits from the hyphae screen and a group of 86 hits from the conidia screen were selected for confirmation in the same assays along with an additional 26 antifungal drugs. A counter-screen using a mammalian SH-SY5Y cell line as a general cytotoxicity assay was also performed in parallel with the confirmation assays to determine the selectivity of these compounds against $E$. rostratum. The anti-E. rostratum activities of 22 compounds were confirmed in the hyphae assay and 37 compounds were confirmed in the conidia assay, with 22 of them being active in both assays. Eight selected compounds from the confirmed compounds were further tested in the traditional colony formation experiment to confirm their anti-E. rostratum activities. doi:10.1371/journal.pone.0070506.g004

for $24 \mathrm{~h}$ at $30^{\circ} \mathrm{C}$ before the detection of cellular viability with the ATP content assay. Screen of conidia was performed in a similar way except that PBS was replaced with RPMI medium and conidia were plated for a final seeding density of 250 conidia/well.

\section{Colony formation}

$10 \mu \mathrm{l}$ of hyphal suspension (processed as described above) was transferred to $1.7 \mathrm{~mL}$ microfuge tubes, each containing $28 \mathrm{nM}$ amphotericin B, $242 \mathrm{nM}$ posaconazole, or $10 \%$ DMSO in $1 \mathrm{ml}$ PBS. Tubes containing hyphae and drug were incubated at $37^{\circ} \mathrm{C}$ in a shaking incubator for $24 \mathrm{~h}$, then $200 \mu \mathrm{l}$ of the hyphae drug suspension was spread on Yeast Extract Peptone Dextrose (YPD) plates for $24 \mathrm{~h}$ at $30^{\circ} \mathrm{C}$ and the number of colony forming units were scored. The experiments were performed in triplicate.

\section{Data analysis}

The primary screen data and curve fitting were analyzed using software developed internally at the NIH Chemical Genomics Center (NGGG) [19]. Total signals for cellular viability $(100 \%$ cellular viability) were calculated from the wells with DMSO solvent and the complete cell killing ( $0 \%$ cellular viability) was calculated from $46 \mu \mathrm{M}$ amphotericin B treated wells. $\mathrm{IC}_{50}$ values of compound confirmation data were calculated using the Prism software (Graphpad Software, Inc. San Diego, CA). All values were expressed as the mean $+/-\mathrm{SD}(\mathrm{n} \geq 3)$.

\section{Results}

Development of fungicidal assays for hyphal fragments and conidia

In standard fungal growth inhibition assays, conidial suspensions are typically used to measure compound activities on the inhibition of fungal growth in a nutrient-rich RPMI medium [20]. Thus, the growth inhibition assay in RPMI medium was adapted for high-throughput screening to identify antifungal compounds (Fig. 1). However, fungal infections may also occur within nutrient-deprived environments such as in the central nervous system or abscess cavities [21]. In addition, many clinical data have indicated that the fungicidal activity of antifungal drugs, exemplified by the polyene amphotericin $\mathrm{B}$, may be more important to clinical success for the treatments of fungal infections [22]. Thus, in addition to the standard growth inhibition assay using conidia, we also developed a hyphal fungicidal assay using nutrient-depleted medium for this drug repurposing screen (Fig. 1). In this study, both hyphae and conidia assays were used for a comprehensive evaluation of therapeutic potentials of antifungal drugs since the current outbreak of infections has been reported in both the CNS and within other closed spaces such as peripheral joints [3].

We used a commercially available ATP content assay kit to measure the viability of hyphal fragments or conidia after treatment with compounds. The bioluminescence signals are generated by the interaction of ATP in lysates of live cellular elements with luciferase and its substrate luciferin added from the assay kit. This assay was suitable for use in 1536-well plates because of its high sensitivity and simple assay procedure. The hyphal fragment density was first optimized using 3750, 7500 and 15000 fragments/well in 1536-well plates with amphotericin B as a positive control. The total ATP content signals in control group (without compound treatment) increased with the increase in hyphal densities while the signals in the group treated with amphotericin B for 24 hours reduced dramatically, indicating almost complete killing of hyphae (Fig. 2a). The signal-to-basal (S/ B) ratio increased from 35 fold to 70 fold when the cellular densities rose from 3500 to 7500-15000 cellular equivalents/well (Fig. 2b). The hyphal fragment cellular density of 15000/well was then selected for further experiments. The conidia density was also optimized in a similar manner. The signals of viable conidia increased with increased cellular densities. We selected 250 conidia/well for subsequent experiments. The $\mathrm{IC}_{50}$ values of amphotericin B were similar in both our 1536-well plate assays (Fig. 3); $12.4 \mathrm{nM}$ in the hyphal assay and $9.41 \mathrm{nM}$ in the conidia assay that were 5 to 6 fold more potent than $60 \mathrm{nM}$ determined from the microdilution method for filamentous fungi [23]. This discrepancy might be due to differences in assay formats as one measures the ATP contents in viable cellular elements and the microdilution method counts viable cell density directly. Fluconazole, another antifungal drug without activity against molds such as E. rostratum, showed no activity at concentrations up to $46 \mu \mathrm{M}$ 


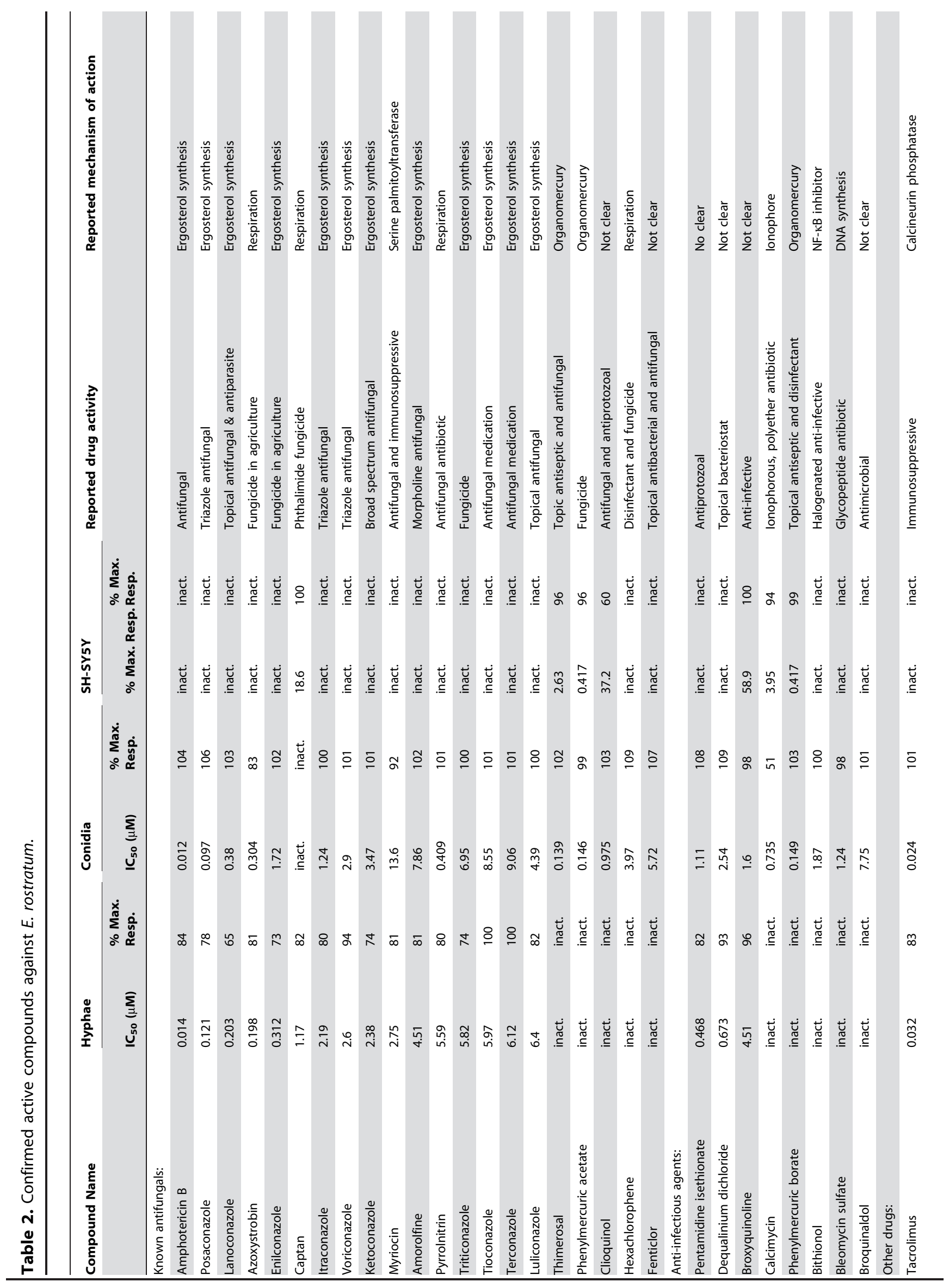




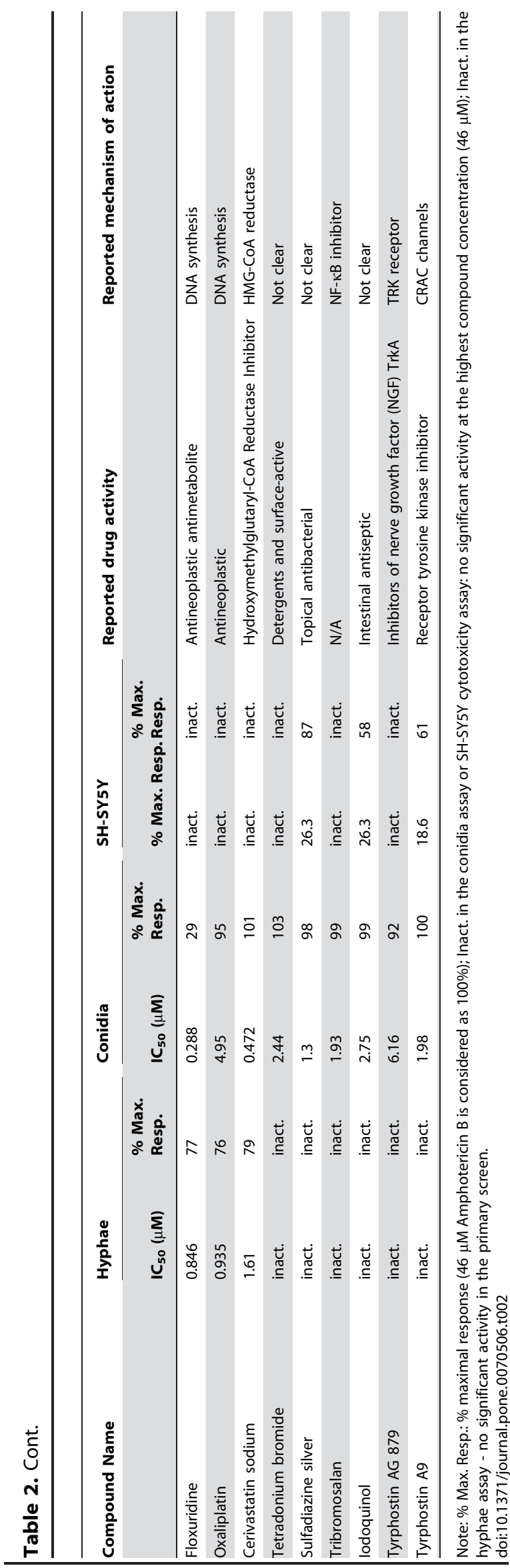

in either the hyphae or conidial assay format (Fig. 3). The results indicate that both hyphae and conidia assays in the ATP content assay format are suitable for compound screening to identify antiE. rostratum agents.

\section{Compound library screening and hit confirmation}

Two small known compound libraries including the LOPAC (1280 compounds) and approved drugs (4096 compounds) were screened in parallel using the above optimized assays for hyphae and conidia in 1536-well plates (Fig. 4). A total of 87 primary hits were identified from the hyphal assay and 86 compounds were found from the conidia screen. These hits were retested in the same assays for their concentration response curves along with 26 additional known antifungal drugs (Table S1). A mammalian SHSY5Y cell line was used in parallel as a counter-screen with the same ATP content assay kit to assess the specificity of these compounds for E. rostratum. As a result, 22 compounds in the hyphal assay and 37 compounds in the conidial assay showed activity against $E$. rostratum with $\mathrm{IC}_{50}$ values under $10 \mu \mathrm{M}$ and with greater than 10-fold selectivity over the SH-SY5Y cells (Table 2). Among these confirmed compounds, 21 of them were active in both hyphal and conidia assays along with one compound active in the hyphal assay only and 16 compounds selective to the conidia assay.

Several triazole antifungal drugs were among the confirmed compounds, including the most potent one - posaconazole (hyphae $\mathrm{IC}_{50}=121 \mathrm{nM}$, conidia $\mathrm{IC}_{50}=97 \mathrm{nM}$ ), as well as lanoconazole (hyphae $\mathrm{IC}_{50}=203 \mathrm{nM}$, conidia $\mathrm{IC}_{50}=380 \mathrm{nM}$ ), voriconazole (hyphae $\mathrm{IC}_{50}=2.64 \mu \mathrm{M}$, conidia $\mathrm{IC}_{50}=2.91 \mu \mathrm{M}$ ), itraconazole (hyphae $\mathrm{IC}_{50}=2.19 \mu \mathrm{M}$, conidia $\mathrm{IC}_{50}=1.24 \mu \mathrm{M}$ ), and luliconazole (hyphae $\mathrm{IC}_{50}=6.40 \mu \mathrm{M}$, conidia $\left.\mathrm{IC}_{50}=4.39 \mu \mathrm{M}\right)$ (Fig. 5, Table 2). The $\mathrm{IC}_{50}$ values were similar to these (posaconazole: $0.25 \mu \mathrm{g} / \mathrm{ml}$ or $360 \mathrm{nM}$; voriconazole: $2 \mu \mathrm{g} / \mathrm{ml}$ or $5.7 \mu \mathrm{M}$ ) determined using the microdilution method (Clinical and Laboratory Standards Institute) for filamentous fungi M38-A2 for this isolate [23].

In addition to known antifungals, several other known drugs were identified as potential anti-E. rostratum agents. Bithionol, an old anti-parasitic drug with central nervous system penetration, was active with an $\mathrm{IC}_{50}$ of $1.87 \mu \mathrm{M}$ in the conidia assay (Table 2). In addition, a number of known drugs with immunosuppressive or anti-cancer properties were also found having anti-E. rostratum acitivies, though they may not be suitable for primary therapy as they could suppress the immune response of patients. However, these drugs may provide prophylactic activity against E. rostratum infection if the patient was on these therapies for other reasons. For example, tacrolimus (Fig. 5D), an immunosuppressive agent used after organ transplantation, exhibited potent activity in both hyphae $\left(\mathrm{IC}_{50}=32 \mathrm{nM}\right)$ and conidia $\left(\mathrm{IC}_{50}=24 \mathrm{nM}\right)$ assays. Another known drug with anti- E. rostratum activity was floxuridine (Table 2), an antimetabolite used to treat a variety of tumors including brain tumors [24]. Its $\mathrm{IC}_{50}$ value in the hyphal assay was $846 \mathrm{nM}$. Floxuridine crosses the blood brain barrier with brain concentrations above $600 \mathrm{nM} 8$ hours after an intravenous (iv) administration of $20 \mathrm{mg} / \mathrm{kg}$ of the drug [25]. Among the 38 confirmed hits (Table 2), many may not be suitable for direct use to treat infections with E. rostratum. Diverse molecular targets and mechanism of action (Fig. 6) have been reported for the 8 'antiinfective agents' and 10 'other agents' listed in Table 2. Taken together, we have identified several non-antifungal drugs that exhibited fungicidal activity against E. rostratum. Since most are approved drugs, they may be used in either single use or in a combination with amphotericin B for the treatment or concomitant prophylaxis of infections caused by E. rostratum. 

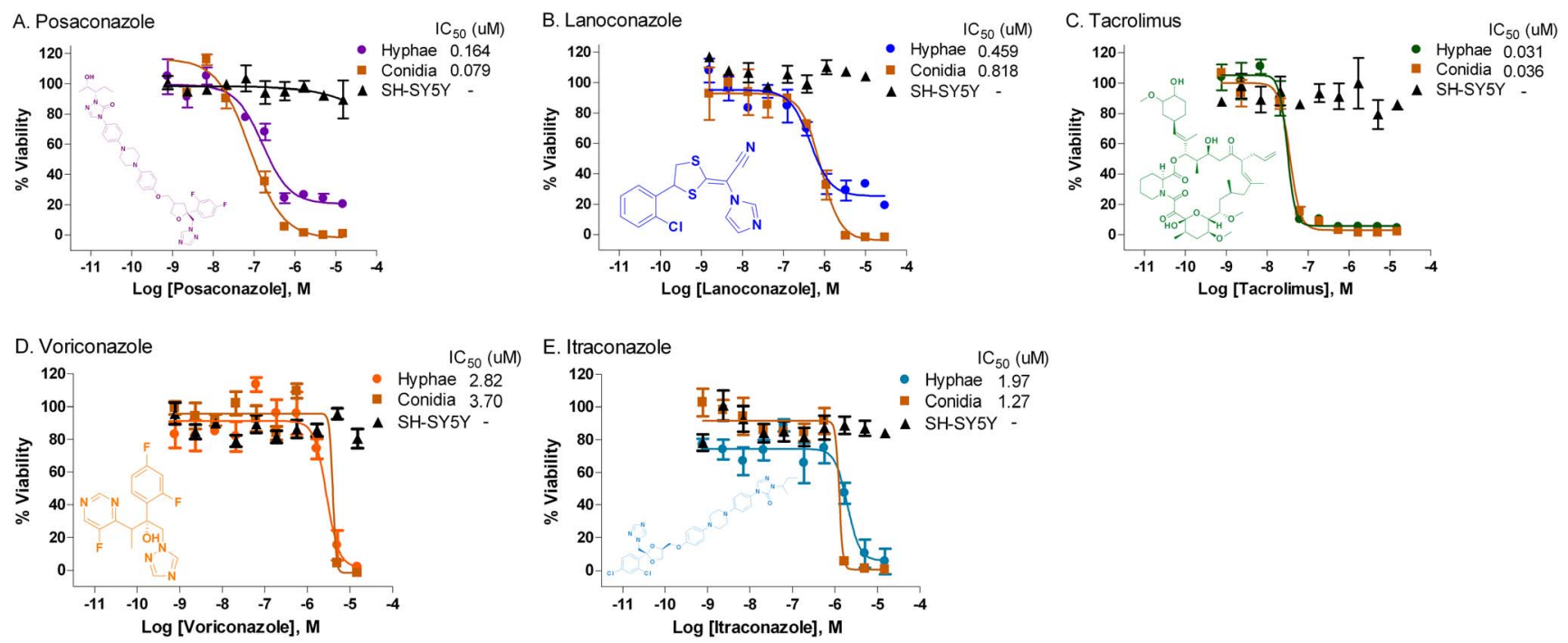

Figure 5. Representative confirmed compounds against $E$. rostratum. The antifungal activities of five compounds including (A) posaconazole, (B) lanoconazole, (C) tacrolimus, (D) voriconazole, and (E) itraconazole were compared in the hyphae and conidia assays with their cytotoxicities in SH-SY5Y mammalian cells. All of these five compounds exhibited similar antifungal activities in both hyphae and conidia assays with no significant cytotoxicity in the mammalian cells at the concentration up to $46 \mu \mathrm{M}$. doi:10.1371/journal.pone.0070506.g005

\section{Confirmation of fungicidal activity in colony formation assays}

To further validate the fungicidal activities of compounds identified from our new assays, we measured viability by colony forming units of E. rostratum hyphal fragments in the presence of amphotericin B, posaconazole, lanoconazole, voriconazole, itraconazole, luliconazole, tacrolimus and floxuridine (Fig. 7). After treatment with these compounds at $37^{\circ} \mathrm{C}$ for $24 \mathrm{~h}$, all the compounds at concentrations of 2 times their respective $\mathrm{IC}_{50}$ values significantly reduced hyphae-derived colony forming units $(\mathrm{GFU})$ compared to that in the DMSO control $(\mathrm{p}<0.01)$. Together, the results further demonstrate the activities of these compounds against E. rostratum.

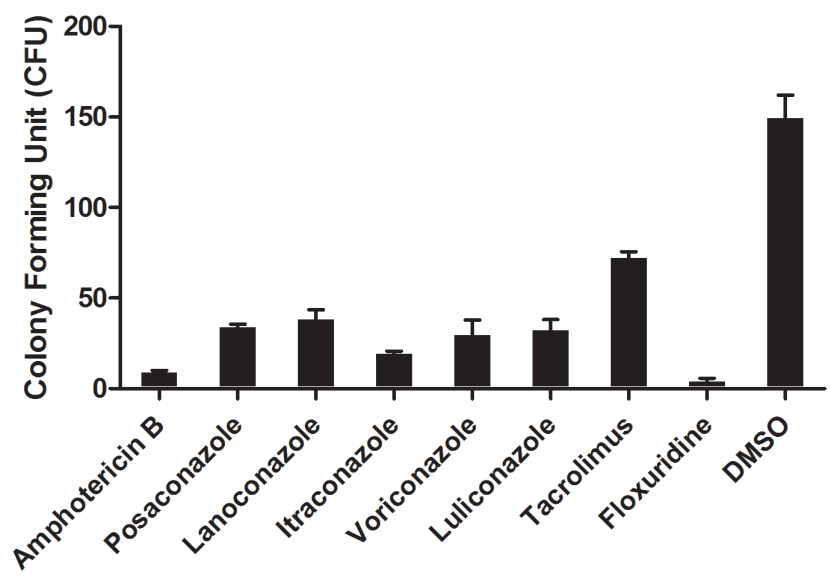

Figure 6. Distribution of molecular targets of 18 confirmed compoundss. The antifungal activities against $E$. rostratum were identified for 8 other anti-infectious agents and 10 other drugs (for details, see Table 2). Their molecular targets and mechanism of action are known although their antifungal activities were not previously reported. These reported molecular targets may implicate potential new directions for anti-E. rostratum drug development. doi:10.1371/journal.pone.0070506.g006

\section{Discussion}

The E. rostratum infection outbreak thus far has resulted in approximately 741 cases with a large number of CNS infections and 55 deaths. Advanced age of the patient population has limited therapeutic applications in some patients and many have responded suboptimally, requiring changes in therapy. The present pilot screen successfully identified several known drugs as alternative therapeutic choices from a collection of known drugs and pharmaceutically active compounds within a seven-week period. The original goal of such a screen was to identify a spectrum of antifungal agents such as posaconazole that could be used immediately to treat patients with E. rostratum infection. We also found a group of novel agents such as bithionol that could be developed further in animal models, preclinical and clinical studies in the event the outbreak continued or drug resistance was encountered. The results from this drug repurposing screen were also helpful in excluding agents that are unlikely to be useful in clinical therapy. For example, albendazole has shown activity against other molds such as Aspergillus [26] but is not active against E. rostratum in our experiments. Therefore, this study demonstrates that such a drug repurposing screen can be conducted within a useful time-frame and should be considered as part of the initial public health response to new outbreaks or emerging microbial pathogens.

To improve assessment of the in vitro activity of antifungal agents against hyphae, quantitative colorimetric assays using 2,3-bis(2methoxy-4-nitro-5-[(sulphenylamino)carbonyl]-2H-tetrazolium-

hydroxide (XTT) and a fluorometric assay using AlarmaBlue have been developed [27]. However, the lower signal-to-basal ratio in either assay limits their applications in high-throughput screening. An alternative cell viability assay that measures cellular ATP levels (termed ATP content assay) with a luminescence readout has been successfully applied in high throughput screening to identify lead compounds for a verity of targets $[10,28,29,30,31]$. ATP is the primary energy storage in all cells and can be used as an important marker for the functional integrity of live cells. The ATP content assay has been used to determine compound cytotoxicity in 


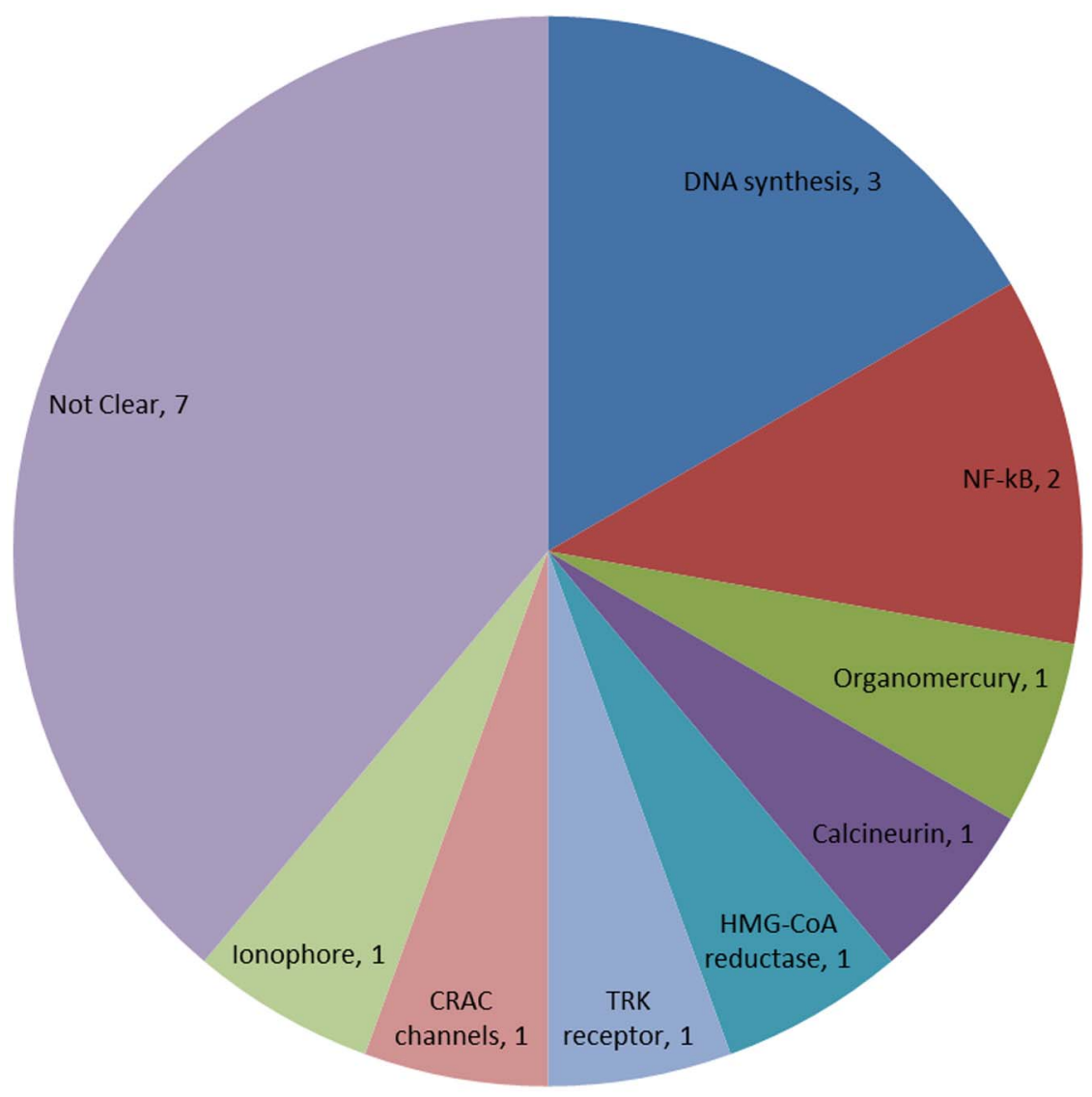

Figure 7. Validation of anti-E. rostratum activities of 8 confirmed compounds in colony forming assay. Hyphal fragment suspensions were treated with either $28 \mathrm{nM}$ amphotericin B, $242 \mathrm{nM}$ posaconazole, $406 \mathrm{nM}$ lanoconazole, $4.38 \mu \mathrm{M}$ itraconazole, $5.2 \mu \mathrm{M}$ voriconazole, $12.8 \mu \mathrm{M}$ luliconazole, $64 \mathrm{nM}$ tacrolimus, $1.692 \mu \mathrm{M}$ floxuridine or $1 \%$ DMSO (a solvent control) in PBS. Mixtures were cultured at $37^{\circ} \mathrm{C}$ in a shaking incubator for $24 \mathrm{~h}$, then the hyphal suspensions were inoculated on Yeast Extract Peptone Dextrose (YPD) plates that were incubated for 1 day. The number of colonies on each YPD plate was counted. Results from three independent experiments were averaged; error bars show standard deviation. doi:10.1371/journal.pone.0070506.g007

mammalian cells and more recently Thielavia subthermophila fungal growth $[32,33,34]$. This assay is a homogenous assay with 'one step' reagent addition in which no cell wash step is required. A luminescence signal is generated upon the release of cellular ATP that is detectable following a 10-minute incubation. A unique feature of the ATP content assay is that the signal-to-basal ratio is characteristically much higher in comparison to the AlamarBlue assay, partly due to low innate luminescence background generated by cells, reagents or plates. Our results have demonstrated that the new assay in the 1536-well plate format can measure the sensitivities of thousands of known drugs against $E$. rostratum in a short period of time that is not possible for conventional antibiotic susceptibility testing (AST). This new high throughput method may also be extended to other fungi or bacteria for identification of useful drugs from all known approved compounds.

Our data indicate that two potent triazole antifungal agents including posaconazole and lanoconazole (under development) may be useful as alternative agents for the treatment of infections with E. rostratum. The high potency and fungicidal activity of posaconazole to $E$. rostratum may suggest a role in the treatment of non-CNS infections such as septic joints that have also featured predominantly in the current outbreak and may allow prolonged oral therapy. Posaconazole has been reported to penetrate the blood brain barrier and may thus be an effective alternative for fungal infections within the central nervous system, though it does not reach reliable CNS levels compared to azoles such as voriconazole, [20].

Bithionol, an old anti-parasitic drug, is not previously known for its antifungal activity. The serum concentration of this drug has been found to be between 0.08 and $0.18 \mathrm{mg} / \mathrm{l}$ during administration and has been used successfully in the treatment of CNS Paragonimus infections $[21,22]$. Thus, it might be a good candidate for the further clinical trials to treat CNS infections of E. rostratum.

Although tacrolimus, an immunosuppressive agent, showed potent activity against E. rostratum in this study, it would not be useful for treatment of infections with $E$. rostratum because the immunosuppression may counteract its fungicidal activity. The mechanism of action of tacrolimus has been reported through inhibition of calcineurin that, in mammalian cells, prevents transport of the nuclear factor of activated $\mathrm{T}$ cells (NF-ATc) to the nucleus and reduces protective IL-2 production [23]. Tacrolimus is a lipophilic agent that may have several neurotoxic effects, especially on lipid-rich white matter which is reversible after dose reduction [24,25]. However, the potent effect of tacrolimus against E. rostratum suggests that anti-calcineurin compounds without these immunosuppressive properties may be of benefit against pigmented molds. This indicates that calcineurin might be a new target for drug development to treat infections with $E$. rostratum.

Floxuridine, an antimetabolite cancer drug, exhibited high nanomolar activity against E. rostratum. However, its cytotoxicity 
would likely prevent its use in treatment of infection with $E$. rostratum. Thus, the structure of this compound requires modification to reduce cytotoxicity and to enhance potency against $E$. rostratum before being applied as a therapeutic drug.

While many of the confirmed hits may not be suitable for treatment of fungal infections, their fungicidal activities may suggest new targets for future drug development after their potencies are optimized. For example, azoxystrobin, captan, pyrrolnitrin and hexachlorophene inhibit respiration $[35,36,37,38]$; myriocin inhibits serine palmitoyltransferase $[39,40]$; and thimerosal and phenylmercuric acetate are toxic organomercuries [41,42]; while clioquinol and fenticlor have unclear mechanisms of action. Bleomycin sulfate, floxuridine and oxaliplatin inhibit DNA synthesis $[43,44,45]$, which suggests that a selective fungal DNA synthesis inhibitor might be developed as a new antifungal agent against molds such as E. rostratum. Tribromosalan and bithionol are nuclear factor-kappa B (NF$\kappa \mathrm{B})$ inhibitors [11]. Phenylmercuric borate is an organomercury $[46,47]$. Tacrolimus binds to the immunophilin FKBP12 to inhibit calcineurin [48]. Cerivastatin sodium is an inhibitor of hydroxymethylglutaryl-coenzyme A (HMG-CoA) reductase [49]. Tyrphostin AG 879 is an inhibitor of tropomyosin-receptor-kinase (TRK) receptor [50]. Tyrphostin A9 is an inhibitor of calcium release-activated calcium (CRAC) channels [51]. Calcimycin is an ionophore [52]. The MOA \& molecular targets of the other 6 compounds are yet to be elucidated. Thus, the data indicate that a rich collection of new drug targets can be identified from such a drug repurposing screen.

\section{References}

1. Kainer MA, Reagan DR, Nguyen DB, Wiese AD, Wise ME, et al. (2012) Fungal infections associated with contaminated methylprednisolone in Tennessee. The New England journal of medicine 367: 2194-2203.

2. Kauffman CA, Pappas PG, Patterson TF (2012) Fungal Infections Associated with Contaminated Methylprednisolone Injections - Preliminary Report. The New England journal of medicine.

3. Smith RM, Schaefer MK, Kainer MA, Wise M, Finks J, et al. (2012) Fungal Infections Associated with Contaminated Methylprednisolone Injections Preliminary Report. The New England journal of medicine.

4. Kerkering TM, Grifasi ML, Baffoe-Bonnie AW, Bansal E, Garner DC, et al. (2013) Early Clinical Observations in Prospectively Followed Patients With Fungal Meningitis Related to Contaminated Epidural Steroid Injections. Annals of internal medicine 158: 154

5. Finks J, Collins J, Miller C, et al. (2013) Spinal and paraspinal infections associated with contaminated methylprednisolone acetate injections - michigan, 2012-2013. MMWR Morbidity and mortality weekly report 62: 377-381.

6. Adler A, Yaniv I, Samra Z, Yacobovich J, Fisher S, et al. (2006) Exserohilum: an emerging human pathogen. European journal of clinical microbiology \& infectious diseases : official publication of the European Society of Clinical Microbiology 25: 247-253.

7. Lin SC, Sun PL, Ju YM, Chan YJ (2009) Cutaneous phaeohyphomycosis caused by Exserohilum rostratum in a patient with cutaneous T-cell lymphoma. International journal of dermatology 48: 295-298.

8. Juhas E, Reyes-Mugica M, Michaels MG, Grunwaldt LJ, Gehris RP (2012) Exserohilum Infection in an Immunocompromised Neonate. Pediatric dermatology.

9. Huang R, Southall N, Wang Y, Yasgar A, Shinn P, et al. (2011) The NCGC pharmaceutical collection: a comprehensive resource of clinically approved drugs enabling repurposing and chemical genomics. Science translational medicine 3: 80ps16.

10. Chen CZ, Kulakova L, Southall N, Marugan JJ, Galkin A, et al. (2011) Highthroughput Giardia lamblia viability assay using bioluminescent ATP content measurements. Antimicrobial agents and chemotherapy 55: 667-675.

11. Miller SC, Huang R, Sakamuru S, Shukla SJ, Attene-Ramos MS, et al. (2010) Identification of known drugs that act as inhibitors of NF-kappaB signaling and their mechanism of action. Biochemical pharmacology 79: 1272-1280.

12. Shen M, Zhang Y, Saba N, Austin CP, Wiestner A, et al. (2013) Identification of Therapeutic Candidates for Chronic Lymphocytic Leukemia from a Library of Approved Drugs. Manuscript in submission.

13. Xia M, Huang R, Sakamuru S, Alcorta D, Cho M, et al. (2013) Identification of repurposed small molecule drugs for chordoma therapy. Cancer Biology and Therapy In press.
In conclusion, we have developed two high throughput assays using conidia and hyphae of E. rostratum in an ATP content assay format. A screen of 4096 compounds from an approved drug library and a pharmaceutically active compound collection using this assay led to identification and confirmation of a group of potential antifungal agents. While several triazole antifungal agents including posaconazole and lanoconazole could be used as alternative therapies for the treatment of infections with $E$. rostratum, the other confirmed compounds may implicate new drug targets for further drug development to improve their selectivity against molds such as E. rostratum. We believe that implementation of such a drug repurposing screen within a 7 week window provides a useful paradigm. It meets the challenges of future public health threats by enabling the rapid identification of potential therapeutic agents for infected patients and for identification of new drug development targets.

\section{Supporting Information}

Table S1 Activities of 26 known antifungal compounds fungicidal for both hyphae and conidia of $E$. rostratum. (DOCX)

\section{Author Contributions}

Conceived and designed the experiments: PRW WZ. Performed the experiments: WS YP AF. Analyzed the data: WS YP AF. Contributed reagents/materials/analysis tools: JAS NS PS JCM KJKC. Wrote the paper: WS YP.

14. Xu M, Liu K, Swaroop M, Porter FD, Sidhu R, et al, (2012) delta-Tocopherol reduces lipid accumulation in Niemann-Pick type C1 and Wolman cholesterol storage disorders. The Journal of biological chemistry 287: 39349-39360.

15. Yuan H, Myers S, Wang J, Zhou D, Woo JA, et al. (2012) Use of reprogrammed cells to identify therapy for respiratory papillomatosis. The New England journal of medicine 367: 1220-1227.

16. Richard JL, Cysewski SJ, Fichtner RE (1971) Harvest and survival of aspergillus fumigatus Fresenius spores. Mycopathologia et mycologia applicata 43: 165168.

17. Masuko T, Minami A, Iwasaki N, Majima T, Nishimura S, et al. (2005) Carbohydrate analysis by a phenol-sulfuric acid method in microplate format. Analytical biochemistry 339: 69-72.

18. Lea WA, Xi J, Jadhav A, Lu L, Austin CP, et al. (2009) A high-throughput approach for identification of novel general anesthetics. PloS one 4: e7150.

19. Wang Y, Jadhav A, Southal N, Huang R, Nguyen DT (2010) A grid algorithm for high throughput fitting of dose-response curve data. Current chemical genomics 4: 57-66.

20. CLSI (2012) Reference method for Broth Dilution Antifungal Susceptibility Testing of Yeasts; Fourth Informational Supplement. CLSI document M27-S4 Wayne: Clinical and Laboratory Standards Institute (CLSI).

21. Diamond RD, Bennett JE (1974) Prognostic Factors in Cryptococcal Meningitis - Study in 111 Cases. Annals of internal medicine 80: 176-181.

22. Lewis JS, Graybill JR (2008) Fungicidal versus fungistatic: what"s in a word? Expert opinion on pharmacotherapy 9: 927-935.

23. National Committee for Clinical Laboratory Standards (2008) Reference Method for Broth Dilution Susceptibility Testing of Filamentous Fungi: Approved Standard-Second Edition. Wayne, PA: Clinical and Laboratory Standards Institute.

24. Nakagawa H, Yamada M, Fukushima M, Shimizu K, Ikenaka K (1998) In vivo study on intrathecal use of 5-fluoro-2 '-deoxysuridine (FdUrd) in meningeal dissemination of malignant tumor. Neurological Surgery 26: 873-879.

25. Wang JX, Sun X, Zhang ZR (2002) Enhanced brain targeting by synthesis of 3 ',5 '-dioctanoyl-5-fluoro-2 '-deoxyuridine and incorporation into solid lipid nanoparticles. European Journal of Pharmaceutics and Biopharmaceutics 54: 285-290.

26. Berthet N, Faure O, Bakri A, Ambroise-Thomas P, Grillot R, et al. (2003) In vitro susceptibility of Aspergillus spp. clinical isolates to albendazole. J Antimicrob Chemother 51: 1419-1422.

27. Rabjohns JLA, Park YD, Dehdashti J, Zheng W, Williamson PR (2013) A High Throughput Screening Assay for Fungicidal Compounds against Cryptococcus neoformans. Journal of Bimolecular Screen In submission.

28. Cho SH, Warit S, Wan B, Hwang CH, Pauli GF, et al. (2007) Low-oxygenrecovery assay for high-throughput screening of compounds against nonreplicat- 
ing Mycobacterium tuberculosis. Antimicrobial agents and chemotherapy 51: 1380-1385.

29. Coconnier-Polter MH, Lievin-Le Moal V, Servin AL (2005) A Lactobacillus acidophilus strain of human gastrointestinal microbiota origin elicits killing of enterovirulent Salmonella enterica Serovar Typhimurium by triggering lethal bacterial membrane damage. Applied and environmental microbiology 71: 6115-6120.

30. Hu G, Hacham M, Waterman SR, Panepinto J, Shin S, et al. (2008) PI3K signaling of autophagy is required for starvation tolerance and virulenceof Cryptococcus neoformans. The Journal of clinical investigation 118: 1186-1197.

31. Kim K, Pollard JM, Norris AJ, McDonald JT, Sun Y, et al. (2009) Highthroughput screening identifies two classes of antibiotics as radioprotectors: tetracyclines and fluoroquinolones. Clinical cancer research : an official journal of the American Association for Cancer Research 15: 7238-7245.

32. Kusari S, Zuhlke S, Kosuth J, Gellarova E, Spiteller M (2009) Light-independent metabolomics of endophytic Thielavia subthermophila provides insight into microbial hypericin biosynthesis. Journal of natural products 72: 1825-1835.

33. Kangas L, Gronroos M, Nieminen AL (1984) Bioluminescence of cellular ATP: a new method for evaluating cytotoxic agents in vitro. Medical biology 62: 338343.

34. Cree IA, Andreotti PE (1997) Measurement of cytotoxicity by ATP-based luminescence assay in primary cell cultures and cell lines. Toxicology in vitro : an international journal published in association with BIBRA 11: 553-556.

35. Esser L, Quinn B, Li YF, Zhang MQ, Elberry M, et al. (2004) Crystallographic studies of quinol oxidation site inhibitors: A modified classification of inhibitors for the cytochrome bc(1) complex. Journal of Molecular Biology 341: 281-302.

36. Rousk J, Demoling LA, Baath E (2009) Contrasting Short-Term Antibiotic Effects on Respiration and Bacterial Growth Compromises the Validity of the Selective Respiratory Inhibition Technique to Distinguish Fungi and Bacteria. Microbial Ecology 58: 75-85.

37. Tripathi RK, Gottlieb D (1969) Mechanism of action of the antifungal antibiotic pyrrolnitrin. Journal of bacteriology 100: 310-318.

38. Frederick JJ, Corner TR, Gerhardt P (1974) Antimicrobial actions of hexachlorophene: inhibition of respiration in Bacillus megaterium. Antimicrobial agents and chemotherapy 6: 712-721.

39. Mivake Y, Kozutsumi Y, Nakamura S, Fujita T, Kawasaki T (1995) Serine Palmitoyltransferase Is the Primary Target of a Sphingosine-Like Immunosuppressant, Isp-1/Myriocin. Biochemical and Biophysical Research Communications 211: 396-403.

40. Yamaji-Hasegawa A, Takahashi A, Tetsuka Y, Senoh Y, Kobayashi T (2005) Fungal metabolite sulfamisterin suppresses sphingolipid synthesis through inhibition of serine palmitoyltransferase. Biochemistry 44: 268-277.
41. Melnick JG, Yurkerwich K, Buccella D, Sattler W, Parkin G (2008) Molecular structures of thimerosal (Merthiolate) and other arylthiolate mercury alkyl compounds. Inorganic Chemistry 47: 6421-6426.

42. Geier J, Lessmann H, Uter W, Schnuch A (2005) Patch testing with phenylmercuric acetate. Contact Dermatitis 53: 117-118.

43. Hecht SM (2000) Bleomycin: New perspectives on the mechanism of action. Journal of natural products 63: 158-168.

44. Murakami Y, Kazuno H, Emura T, Tsujimoto H, Suzuki N, et al. (2000) Different mechanisms of acquired resistance to fluorinated pyrimidines in human colorectal cancer cells. International Journal of Oncology 17: 277-283.

45. Graham J, Muhsin M, Kirkpatrick P (2004) Oxaliplatin. Nature Reviews Drug Discovery 3: 11-12.

46. Cortat M (1978) Studies on the mode of action of phenylmercuric borate on Escherichia coli. II. Biochemical localization and inhibition of some metabolic activities. Zentralblatt fur Bakteriologie, Parasitenkunde, Infektionskrankheiten und Hygiene Erste Abteilung Originale Reihe B: Hygiene, praventive Medizin 166: 528-539

47. Cortat M (1978) Studies on the mode of action of phenylmercuric borate on Escherichia coli. I. Structural localization and kinetics of incorporation. Zentralblatt fur Bakteriologie, Parasitenkunde, Infektionskrankheiten und Hygiene Erste Abteilung Originale Reihe B: Hygiene, praventive Medizin 166: $517-527$

48. Liu J, Farmer JD, Lane WS, Friedman J, Weissman I, et al. (1991) Calcineurin Is a Common Target of Cyclophilin-Cyclosporine-a and Fkbp-Fk506 Complexes. Cell 66: 807-815.

49. Ganne F, Vasse M, Beaudeux JL, Peynet J, Francois A, et al. (2000) Cerivastatin, an inhibitor of HMG-CoA reductase, inhibits urokinase/urokinase-receptor expression and MMP-9 secretion by peripheral blood monocytes - A possible protective mechanism against atherothrombosis. Thrombosis and Haemostasis 84: 680-688.

50. Ohmichi M, Pang L, Ribon V, Gazit A, Levitzki A, et al. (1993) The Tyrosine Kinase Inhibitor Tyrphostin Blocks the Cellular Actions of Nerve GrowthFactor. Biochemistry 32: 4650-4658.

51. Denys A, Aires V, Hichami A, Khan NA (2004) Thapsigargin-stimulated MAP kinase phosphorylation via CRAC channels and PLD activation: inhibitory action of docosahexaenoic acid. Febs Letters 564: 177-182.

52. Abbott BJ, Fukuda DS, Dorman DE, Occolowitz JL, Debono M, et al. (1979) Microbial Transformation of A23187, a Divalent-Cation Ionophore Antibiotic. Antimicrobial agents and chemotherapy 16: 808-812. 